\title{
A uniform estimate for rate functions in large deviations
}

\section{Luchezar Stoyanov ${ }^{1}$}

\begin{abstract}
Given Hölder continuous functions $f$ and $\psi$ on a subshift of finite type $\Sigma_{A}^{+}$ such that $\psi$ is not cohomologous to a constant, the classical large deviation principle holds with a rate function $I_{\psi} \geqslant 0$ such that $I_{\psi}(p)=0$ iff $p=\int \psi d \mu$, where $\mu=\mu_{f}$ is the equilibrium state of $f$. In this paper we derive a uniform estimate from below for $I_{\psi}$ for $p$ outside an interval containing $\widetilde{\psi}=\int \psi d \mu$, which depends only on the subshift $\Sigma_{A}^{+}$, the function $f$, the norm $|\psi|_{\infty}$, the Hölder constant of $\psi$ and the integral $\widetilde{\psi}$. Similar results can be derived in the same way, e.g. for Axiom A diffeomorphisms on basic sets.
\end{abstract}

Keywords Large deviations · Rate function - Subshift of finite type · Equilibrium state

Mathematics Subject Classification 37A05 $\cdot$ 37B10 $\cdot$ 37D20

\section{Introduction}

Let $T: X \rightarrow X$ be a transformation preserving an ergodic probability measure $\mu$ on a set $X$. Given an observable $\psi: X \rightarrow \mathbb{R}$, Birkhoff's ergodic theorem implies that

$$
\frac{\psi_{n}(x)}{n}=\frac{\psi(x)+\psi(T(x))+\cdots+\psi\left(T^{n-1}(x)\right)}{n} \rightarrow \int_{X} \psi d \mu
$$

$凶$ Luchezar Stoyanov

luchezar.stoyanov@uwa.edu.au

1 School of Mathematics and Statistics, University of Western Australia, 35 Stirling Hwy, Crawley, Perth, WA 6009, Australia 
for $\mu$-almost all $x \in X$. It follows from general large deviation principles (see $[3,6,12]$ ) that if $X$ is a mixing basic set for an Axiom A diffeomorphism $T$, and $f$ and $\psi$ are Hölder continuous functions on $X$ with equilibrium states $\mu=\mu_{f}$ and $\mu_{\psi}$, respectively, and $\psi$ is not cohomologous to a constant (see the definition below), then there exists a real-analytic rate function $I=I_{\psi}: \operatorname{Int}\left(\mathcal{J}_{\psi}\right) \rightarrow[0, \infty)$, where $\mathcal{J}_{\psi}=\left\{\int \psi d m: m \in \mathcal{M}_{T}\right\}$, such that

$$
\lim _{\delta \rightarrow 0} \lim _{n \rightarrow \infty} \frac{1}{n} \log \mu\left(\left\{x \in X: \frac{\psi_{n}(x)}{n} \in(p-\delta, p+\delta)\right\}\right)=-I_{\psi}(p)
$$

for all $p \in \operatorname{Int}\left(\mathcal{J}_{\psi}\right)$. Here $\mathcal{M}_{T}$ is the set of all $T$-invariant Borel probability measures on $X$. Moreover, $I(p)=0$ if and only if $p=\int \psi d \mu$, and the (closed) interval $\mathcal{J}_{\psi}$ is non-trivial, since $\psi$ is not cohomologous to a constant.

Similar large deviation principles apply for any subshift of finite type $\sigma: \Sigma_{A}^{+} \rightarrow \Sigma_{A}^{+}$ on a one-sided shift space

$$
\Sigma_{A}^{+}=\left\{\xi=\left(\xi_{0}, \xi_{1}, \ldots, \xi_{m}, \ldots\right): 1 \leqslant \xi_{i} \leqslant s_{0}, A\left(\xi_{i}, \xi_{i+1}\right)=1 \text { for all } i \geqslant 0\right\}
$$

Here $A$ is an $s_{0} \times s_{0}$-matrix of 0 's and 1's $\left(s_{0} \geqslant 2\right)$. We assume that $A$ is aperiodic, i.e. there exists an integer $M>0$ such that $A^{M}(i, j)>0$ for all $i, j$ (see, e.g. [7, Chapter 1]). The shift map $\sigma$ is defined by $\sigma(\xi)=\xi^{\prime}$, where $\xi_{i}^{\prime}=\xi_{i+1}$ for all $i \geqslant 0$. We consider $\Sigma_{A}^{+}$with a metric $d_{\theta}$ defined for some constant $\theta \in(0,1)$ by $d_{\theta}(\xi, \eta)=0$ if $\xi=\eta$ and $d_{\theta}(\xi, \eta)=\theta^{k}$ if $\xi \neq \eta$ and $k \geqslant 0$ is the maximal integer with $\xi_{i}=\eta_{i}$ for $0 \leqslant i \leqslant k$.

For any function $g: \Sigma_{A}^{+} \rightarrow \mathbb{R}$ set

$$
\begin{array}{ll}
\operatorname{var}_{k} g=\sup \left\{|g(\xi)-g(\eta)|: \xi_{i}=\eta_{i}, 0 \leqslant i \leqslant k\right\}, & |g|_{\theta}=\sup \left\{\frac{\operatorname{var}_{k} g}{\theta^{k}}: k \geqslant 0\right\}, \\
|g|_{\infty}=\sup \left\{|g(\xi)|: \xi \in \Sigma_{A}^{+}\right\}, & \|g\|_{\theta}=|g|_{\theta}+|g|_{\infty} .
\end{array}
$$

Denote by $\mathcal{F}_{\theta}\left(\Sigma_{A}^{+}\right)$the space of all functions $g$ on $\Sigma_{A}^{+}$with $\|g\|_{\theta}<\infty$.

Two functions $f, g$ on $\mathcal{F}_{\theta}\left(\Sigma_{A}^{+}\right)$are called cohomologous if there exists a continuous function $h$ on $\Sigma_{A}^{+}$such that $f=g+h \circ \sigma-h$.

The Ruelle transfer operator $L_{f}: C\left(\Sigma_{A}^{+}\right) \rightarrow C\left(\Sigma_{A}^{+}\right)$is defined by

$$
L_{f} g(x)=\sum_{\sigma(y)=x} e^{f(y)} g(y)
$$

Here $C\left(\Sigma_{A}^{+}\right)$denotes the space of all continuous functions $g: \Sigma_{A}^{+} \rightarrow \mathbb{R}$ with respect to the metric $d_{\theta}$. Denote by $\operatorname{Pr}(\psi)$ the topological pressure

$$
\operatorname{Pr}(\psi)=\sup _{m \in \mathcal{M}_{\sigma}}\left(h_{\sigma}(m)+\int \psi d m\right)
$$


of $\psi$ with respect to the map $\sigma$, where $\mathcal{M}_{\sigma}$ is the set of all $\sigma$-invariant probability measures on $\Sigma_{A}^{+}$and $h_{\sigma}(m)$ is the measure theoretic entropy of $m$ with respect to $\sigma$ (see [7] or [10]). Given $\psi \in \mathcal{F}_{\theta}\left(\Sigma_{A}^{+}\right)$, there exists a unique $\sigma$-invariant probability measure $\mu_{\psi}$ on $\Sigma_{A}^{+}$such that

$$
\operatorname{Pr}(\psi)=h_{\sigma}\left(\mu_{\psi}\right)+\int \psi d \mu_{\psi}
$$

(see, e.g. [7, Theorem 3.5]). The measure $\mu_{\psi}$ is called the equilibrium state of $\psi$.

For brevity throughout we write $\int h d m$ for $\int_{\Sigma_{A}^{+}} h d m$. In what follows we assume that $\theta \in(0,1)$ is a fixed constant, $f: \Sigma_{A}^{+} \rightarrow \mathbb{R}$ is a fixed function in $\mathcal{F}_{\theta}\left(\Sigma_{A}^{+}\right)$and $\mu=\mu_{f}$.

As we mentioned earlier, it follows from the Large Deviation Theorem $[3,6,12]$ that if $\psi$ is not cohomologous to a constant, then there exists a real analytic rate function $I=I_{\psi}: \operatorname{Int}\left(\mathcal{J}_{\psi}\right) \rightarrow[0, \infty)$ with $I(p)=0$ iff $p=\int \psi d \mu$ for which (1) holds. More precisely, we have

$$
-I(p)=\inf \{\operatorname{Pr}(f+q \psi)-\operatorname{Pr}(f)-q p: q \in \mathbb{R}\}
$$

It is also known that

$$
\left[\frac{d}{d q} \operatorname{Pr}(f+q \psi)\right]_{q=\eta}=\int \psi d \mu_{f+\eta \psi},
$$

and $\operatorname{Pr}(f+q \psi)$ is a strictly convex function of $q$ (see $[7,10]$ or [4]).

In his paper we derive an estimate from below for $I_{\psi}(p)$ for $p$ outside an interval containing

$$
\widetilde{\psi}=\int \psi d \mu
$$

The estimate depends only on $|\psi|_{\infty}, \widetilde{\psi},|\psi|_{\theta}$ and some constants determined by the given function $f$. In what follows we use the notation $\min \psi=\min _{x \in \Sigma_{A}^{+}} \psi(x)$,

$$
b=b_{\psi}=\max \left\{1,|\psi|_{\theta}\right\}, \quad B_{\psi}=\widetilde{\psi}-\min \{0, \min \psi\} .
$$

Since $\widetilde{\psi}>\min \psi(\psi$ is not cohomologous to a constant), we have $\widetilde{\psi}-\min \psi>0$, so $B_{\psi}>0$ always.

Theorem 1.1 Let $f, \psi \in \mathcal{F}_{\theta}\left(\Sigma_{A}^{+}\right)$be real-valued functions. Assume that $\psi$ is not cohomologous to a constant, and let $0<\delta_{0}<B_{\psi}$. Then for all $p \notin\left[\widetilde{\psi}-\delta_{0}, \widetilde{\psi}+\delta_{0}\right]$ we have

$$
I_{\psi}(p) \geqslant \frac{\delta_{0} q_{0}}{2}
$$


where $q_{0}=\min \{C, 1 / b\}$ for some constant $C>0$ depending only on $|f-\operatorname{Pr}(f)|_{\infty}$, $|f|_{\theta},|\psi|_{\infty}, \widetilde{\psi}$ and $\delta_{0}$.

The motivation to try to obtain estimates of the kind presented in Theorem 1.1 comes from attempts to get some kind of an 'approximate large deviation principle' for characteristic functions $\chi_{K}$ of arbitrary compact sets $K$ of positive measure. In the special case when the boundary $\partial K$ of $K$ is 'relatively regular' (e.g. $\mu(\partial K)=0$ ) large deviation results were established by Leplaideur and Saussol in [5], and also by Kachurovskii and Podvigin [2]. The next example presents a first step in the case of an arbitrary compact set $K$ of positive measure.

Example 1.2 Let $K$ be a compact subset of $\Sigma_{A}^{+}$with $0<\mu(K)<1$, let $0<\delta_{0} \leqslant$ $\mu(K)$, and let $\psi$ be a Hölder continuous function that approximates $\chi_{K}$ from above, i.e. $0 \leqslant \psi \leqslant 1, \psi=1$ on $K$ and $\psi=0$ outside a small neighbourhood $V$ of $K$. Then $b=|\psi|_{\theta} \gg 1$ if $V$ is sufficiently small, so $q_{0}$ in Theorem 1.1 has the form $q_{0}=1 / b$. It then follows from Theorem 1.1 (in fact, from Lemma 2.3) that $I_{\psi}(p) \geqslant \delta_{0} /\left(2|\psi|_{\theta}\right)$ for $p \notin\left[\widetilde{\psi}-\delta_{0}, \widetilde{\psi}+\delta_{0}\right]$.

A result similar to Theorem 1.1 can be stated, e.g. for Axiom A diffeomorphisms on basic sets. Recall that if $F: M \rightarrow M$ is a $C^{1}$ Axiom A diffeomorphism on a Riemannian manifold $M$, a non-empty subset $\Lambda$ of $M$ is called a basic set for $F$ if $\Lambda$ is a locally maximal compact $F$-invariant subset of $M$ which is not a single orbit, $F$ is hyperbolic and transitive on $\Lambda$, and the periodic points of $F$ in $\Lambda$ are dense in $\Lambda$ (see, e.g. [1] or [7, Appendix III]). It follows from the existence of Markov partitions that there exists a two-sided subshift of finite type $\sigma: \Sigma_{A} \rightarrow \Sigma_{A}$ and a continuous surjective map $\pi: \Sigma_{A} \rightarrow \Lambda$ such that: (i) $F \circ \pi=\pi \circ \sigma$, and (ii) for every Hölder continuous function $g$ on $\Lambda, f=g \circ \pi \in \mathcal{F}_{\theta}$ for some $\theta \in(0,1)$ and $\pi$ is oneto-one almost everywhere with respect to the equilibrium state of $f$. Given a Hölder continuous function $g$ on $\Lambda$, the rate function $I_{g}$ is naturally related to the rate function $I_{f}$ of $f=g \circ \pi$. On the other hand, $f$ is cohomologous to a function $f^{\prime} \in \mathcal{F}_{\sqrt{\theta}}\left(\Sigma_{A}\right)$ which depends on forward coordinates only, so $f^{\prime} \in \mathcal{F}_{\sqrt{\theta}}\left(\Sigma_{A}^{+}\right)$. Applying Theorem 1.1 to $f^{\prime}$ provides a similar result for $f$ and therefore for $g$.

For some hyperbolic systems, large deviation principles similar to (1), however with shrinking intervals, have been established recently in $[8,9]$.

\section{Proof of Theorem 1.1}

\subsection{The Ruelle-Perron-Frobenius Theorem}

For convenience of the reader we state here a part of the estimates in [11] that will be used in this section.

Theorem 2.1 (Ruelle-Perron-Frobenius) Let the $s_{0} \times s_{0}$-matrix $A$ and $M>0$ be as in Sect. 1, let $f \in \mathcal{F}_{\theta}\left(\Sigma_{A}^{+}\right)$be real-valued, and let $b_{f}=\max \left\{1,|f|_{\theta}\right\}$. Then:

(i) There exist a unique $\lambda=\lambda_{f}>0$, a probability measure $v=v_{f}$ on $\Sigma_{A}^{+}$and a positive function $h=h_{f} \in \mathcal{F}_{\theta}\left(\Sigma_{A}^{+}\right)$such that $L_{f} h=\lambda h$ and $\int h d v=1$. The 
spectral radius of $L_{f}$ as an operator on $\mathcal{F}_{\theta}\left(\Sigma_{A}^{+}\right)$is $\lambda$, and its essential spectral radius is $\theta \lambda$. The eigenfunction $h$ satisfies

$$
\|h\|_{\theta} \leqslant \frac{6 s_{0}^{M} b_{f}}{\theta^{2}(1-\theta)} e^{4 b_{f} /(1-\theta)} e^{2 M|f|_{\infty}}, \quad \min h \geqslant \frac{1}{e^{2 b_{f} /(1-\theta)} s_{0}^{M} e^{2 M|f|_{\infty}}}
$$

Moreover,

$$
\frac{\min h}{|h|_{\infty}} \lambda^{n} \leqslant L_{f}^{n} 1 \leqslant \frac{|h|_{\infty}}{\min h} \lambda^{n}
$$

for any integer $n \geqslant 0$.

(ii) The probability measure $\widehat{v}=h v$ (this is the so-called equilibrium state of $f$ ) is $\sigma$-invariant and $\widehat{v}=v_{\widehat{f}}$, where $\widehat{f}=f-\log (h \circ \sigma)+\log h-\log \lambda$. Moreover $L_{\widehat{f}} 1=1$.

(iii) For every $g \in \mathcal{F}_{\theta}\left(\Sigma_{A}^{+}\right)$and every integer $n \geqslant 0$ we have

$$
\left\|\frac{1}{\lambda^{n}} L_{f}^{n} g-h \int g d v\right\|_{\theta} \leqslant D \rho^{n}\|g\|_{\theta},
$$

where we can take

$$
\rho=\left(1-\frac{1-\theta}{4 s_{0}^{2 M} e^{8 \theta b_{f} /(1-\theta)} e^{4 M|f|_{\infty}}}\right)^{1 / 2 M} \in(0,1)
$$

and

$$
D=10^{8} \frac{b_{f}^{7}}{\theta^{10}(1-\theta)^{8}} s_{0}^{17 M} e^{40 b_{f} /(1-\theta)} e^{33 M|f|_{\infty}}
$$

Remark 2.2 The constants that appear in the above estimates are not optimal. The proof of [11, Theorem 2] follows that in [1, Section 1.B] with a more careful analysis of the estimates involved. The main point here is that, apart from their obvious dependence on parameters related to the subshift of finite type $\sigma: \Sigma_{A}^{+} \rightarrow \Sigma_{A}^{+}$, these constants can be taken to depend only on $|f|_{\theta}$ and $|f|_{\infty}$.

\subsection{Reductions}

Let $f \in \mathcal{F}_{\theta}\left(\Sigma_{A}^{+}\right)$be the fixed function from Sect. 1 and let $\mu=\mu_{f}$ be as before. It follows from the properties of pressure (see, e.g. [10] or [7]) that $\operatorname{Pr}(g+c)=\operatorname{Pr}(g)+c$ for every continuous function $g$ and every constant $c \in \mathbb{R}$. Thus, replacing $f$ by $f-\operatorname{Pr}(f)$, we may assume that $\operatorname{Pr}(f)=0$. Moreover, if $g$ and $h$ are cohomologous continuous functions on $\Sigma_{A}^{+}$, then $\operatorname{Pr}(g)=\operatorname{Pr}(h)$ and the equilibrium states $\mu_{g}$ of $g$ and $\mu_{h}$ of $h$ on $\Sigma_{A}^{+}$coincide. Since $f$ is cohomologous to a function $\phi \in \mathcal{F}_{\theta}\left(\Sigma_{A}^{+}\right)$with $L_{\phi} 1=1$ (see, e.g. [7]), it is enough to prove the main result with $f$ replaced by such 
a function $\phi$. Moreover, $|\phi|_{\infty}$ and $|\phi|_{\theta}$ can be estimated by means of $|f-\operatorname{Pr}(f)|_{\infty}$ and $|f|_{\theta}$ [see e.g. Theorem 2.1 (ii)].

So, from now on we will assume that $L_{\phi} 1=1$. It then follows that $\operatorname{Pr}(\phi)=0$. Let $\mu=\mu_{\phi}$ be the equilibrium state of $\phi$ on $\Sigma_{A}^{+}$.

For the proof of Theorem 1.1 we may assume that $\psi \geqslant 0$. Indeed, assuming the statement of the theorem is true in this case, suppose $\psi$ takes negative values. Set $\psi_{1}=\psi+c$, where $c=-\min \psi$. Then $\psi_{1} \geqslant 0$. Moreover, $\widetilde{\psi}_{1}=\int \psi_{1} d \mu=\widetilde{\psi}+c$, $B_{\psi_{1}}=B_{\psi}$, and for $p_{1}=p+c$ we have

$$
\begin{aligned}
\Gamma_{1}(q) & =p_{1} q-\operatorname{Pr}\left(\phi+q \psi_{1}\right) \\
& =(p+c) q-\operatorname{Pr}(\phi+q \psi+q c)=p q-\operatorname{Pr}(\phi+q \psi)=\Gamma(q)
\end{aligned}
$$

for all $q \in \mathbb{R}$. Therefore (2) implies

$$
\begin{aligned}
I_{\psi}(p) & =\sup \{p q-\operatorname{Pr}(\phi+q \psi): q \in \mathbb{R}\} \\
& =\sup \left\{p_{1} q-\operatorname{Pr}\left(\phi+q \psi_{1}\right): q \in \mathbb{R}\right\}=I_{\psi_{1}}\left(p_{1}\right) .
\end{aligned}
$$

Moreover, if $0<\delta_{0}<B_{\psi}=B_{\psi_{1}}$, then $p \notin\left[\widetilde{\psi}-\delta_{0}, \widetilde{\psi}+\delta_{0}\right]$ is equivalent to $p_{1}=p+c \notin\left[\widetilde{\psi}_{1}-\delta_{0}, \widetilde{\psi}_{1}+\delta_{0}\right]$. Since $\left|\psi_{1}\right|_{\theta}=|\psi|_{\theta}$ and $\left|\psi_{1}\right|_{\infty} \leqslant 2|\psi|_{\infty}$, using Theorem 1.1 for $I_{\psi_{1}}\left(p_{1}\right)$ and changing appropriately the value of the constant $q_{0}$, we get a similar estimate for $I_{\psi}(p)$.

\subsection{Proof of Theorem 1.1 for $\psi \geqslant 0$}

From now on we will assume that $\phi, \psi \in \mathcal{F}_{\theta}\left(\Sigma_{A}^{+}\right)$are fixed real-valued functions such that $\psi \geqslant 0, \psi$ is not cohomologous to a constant, and

$$
L_{\phi} 1=1 .
$$

Given any $q \in \mathbb{R}$, set

$$
f_{q}=\phi+q \psi, \quad L_{q}=L_{f_{q}} .
$$

In what follows we will assume

$$
|q| \leqslant q_{0} \leqslant \frac{1}{b}
$$

for some constant $q_{0}>0$ which will be chosen below. Then $\left|f_{q}\right|_{\theta} \leqslant|\phi|_{\theta}+1$ for all $q$ with (5), and also $\left|f_{q}\right|_{\infty} \leqslant|\phi|_{\infty}+|\psi|_{\infty}$. Thus, setting

$$
C_{0}=\|\phi\|_{\theta}+2 \max \left\{|\psi|_{\infty}, 1\right\} \geqslant 1,
$$

we have

$$
\left\|f_{q}\right\|_{\theta} \leqslant C_{0}, \quad|q| \in\left[0, q_{0}\right]
$$


Let $v_{q}$ be the probability measure on $\Sigma_{A}^{+}$with

$$
L_{q}^{*} v_{q}=\lambda_{q} v_{q}
$$

where $\lambda_{q}$ is the maximal eigenvalue of $L_{q}=L_{f_{q}}$, and let $h_{q}>0$ be a corresponding normalised eigenfunction, i.e. $h_{q} \in \mathcal{F}_{\theta}\left(\Sigma_{A}^{+}\right), L_{q} h_{q}=\lambda_{q} h_{q}$ and $\int h_{q} d v_{q}=1$. Then $\mu_{q}=h_{q} v_{q}$ is the equilibrium state of $f_{q}$, i.e. $\mu_{q}=\mu_{\phi+q \psi}$. Clearly $h_{0}=1$ and $\mu_{0}=\mu$.

Using the uniform estimates in Theorem 2.1, it follows from (6) that there exist constants $D \geqslant 1$ and $\rho \in(0,1)$, depending on $C_{0}$ but not on $q_{0}$, such that

$$
\left\|\frac{1}{\lambda_{q}^{n}} L_{q}^{n} g-h_{q} \int g d v_{q}\right\|_{\theta} \leqslant D \rho^{n}\|g\|_{\theta}
$$

for all integers $n \geqslant 0$, all functions $g \in \mathcal{F}_{\theta}\left(\Sigma_{A}^{+}\right)$and all $q$ with $|q| \in\left[0, q_{0}\right]$.

Set $L=L_{\phi}$. Given $x \in \Sigma_{A}^{+}$and $m \geqslant 0$, set $g_{m}(x)=g(x)+g(\sigma x)+\cdots+$ $g\left(\sigma^{m-1} x\right)$.

It follows from (7) with $g=1$ that $\lambda_{q}=\int L_{q} 1 d \nu_{q}$. Now

$$
\left(L_{q} 1\right)(x)=\sum_{\sigma y=x} e^{f_{q}(y)}=\sum_{\sigma y=x} e^{\phi(y)+q \psi(y)} \leqslant e^{q_{0}|\psi|_{\infty}}(L 1)(x)=e^{q_{0}|\psi|_{\infty}}
$$

for all $x \in \Sigma_{A}^{+}$implies $\lambda_{q} \leqslant e^{q_{0}|\psi|_{\infty}}$. Similarly, $\lambda_{q} \geqslant e^{-q_{0}|\psi|_{\infty}}$. Thus,

$$
e^{-q_{0} C_{0}} \leqslant \lambda_{q} \leqslant e^{q_{0} C_{0}}, \quad|q| \leqslant q_{0} .
$$

To estimate $h_{q}$ for $q$ with (5), first use (8) with $g=1$ to get

$$
\left\|\frac{1}{\lambda_{q}^{n}} L_{q}^{n} 1-h_{q}\right\|_{\theta} \leqslant D \rho^{n}
$$

Using (4), this gives

$$
\begin{aligned}
h_{q}(x) & \leqslant \frac{\left(L_{q}^{n} 1\right)(x)}{\lambda_{q}^{n}}+D \rho^{n}=\frac{1}{\lambda_{q}^{n}} \sum_{\sigma^{n} y=x} e^{(\phi+q \psi)_{n}(y)}+D \rho^{n} \\
& \leqslant \frac{e^{q_{0} C_{0} n}}{\lambda_{q}^{n}}(L 1)(x)+D \rho^{n} \leqslant e^{2 q_{0} C_{0} n}+D \rho^{n}
\end{aligned}
$$

for all $x \in \Sigma_{A}^{+}$and $n \geqslant 0$. Similarly,

$$
h_{q} \geqslant \frac{e^{-q_{0} C_{0} n}}{\lambda_{q}^{n}}(L 1)-D \rho^{n} \geqslant e^{-2 q_{0} C_{0} n}-D \rho^{n}
$$


for all $n \geqslant 0$. Thus,

$$
\max \left\{0, e^{-2 q_{0} C_{0} n}-D \rho^{n}\right\} \leqslant h_{q} \leqslant e^{2 q_{0} C_{0} n}+D \rho^{n}, \quad n \geqslant 0, \quad|q| \leqslant q_{0} .
$$

From now on we will assume that $p \notin\left[\widetilde{\psi}-\delta_{0}, \widetilde{\psi}+\delta_{0}\right]$ is fixed. Consider the function

$$
\Gamma(q)=p q-\operatorname{Pr}(\phi+q \psi), \quad q \in \mathbb{R} .
$$

Then $I(p)=\sup _{q \in \mathbb{R}} \Gamma(q)$. Clearly, $\Gamma(0)=0$ and moreover by (3),

$$
\Gamma^{\prime}(q)=p-\int \psi d \mu_{\phi+q \psi}
$$

In particular, $\Gamma^{\prime}(0)=p-\widetilde{\psi} \notin\left[-\delta_{0}, \delta_{0}\right]$.

We will now estimate the integral in the right-hand side of (11). Let $\alpha>0$ be the constant so that $\rho_{1}=\max \{\rho, \theta\}=e^{-\alpha}$.

Lemma 2.3 Assume that $\psi \geqslant 0$ on $\Sigma_{A}^{+}$and $0<\delta_{0}<B_{\psi}=\widetilde{\psi}$. Set

$$
q_{0}=\min \left\{\frac{\delta_{0}}{100 C_{0}^{2} n_{0}}, \frac{1}{b}\right\},
$$

where $n_{0}$ is the integer with

$$
n_{0}-1 \leqslant \frac{1}{\alpha}\left|\log \frac{\delta_{0}}{16 C_{0} D}\right|<n_{0} .
$$

Then $\Gamma\left(q_{0}\right) \geqslant \delta_{0} q_{0} / 2$ and $\Gamma\left(-q_{0}\right) \geqslant \delta_{0} q_{0} / 2$.

Proof For any $q \in\left[0, q_{0}\right]$ and any integer $n \geqslant 0,(7),(9)$ and (10) yield

$$
\begin{aligned}
\int \psi d \mu_{q} & =\int \psi h_{q} d v_{q}=\frac{1}{\lambda_{q}^{n}} \int L_{q}^{n}\left(\psi h_{q}\right) d v_{q} \\
& =\frac{1}{\lambda_{q}^{n}} \int \sum_{\sigma^{n} y=x} e^{(\phi+q \psi)_{n}(y)} \psi(y) h_{q}(y) d v_{q}(x) \\
& \leqslant e^{2 q_{0} C_{0} n}\left(e^{2 q C_{0} n}+D \rho^{n}\right) \int L^{n} \psi d v_{q} .
\end{aligned}
$$

It follows from (8) with $q=0$ and $g=\psi$ and the choice of $C_{0}$ that

$$
\left|L^{n} \psi-\int \psi d \mu\right|=\left|L^{n} \psi-\int \psi d \nu\right| \leqslant D \rho^{n} C_{0},
$$

therefore $L^{n} \psi \leqslant \widetilde{\psi}+C_{0} D \rho^{n}$. Combining this with the above gives

$$
\int \psi d \mu_{q} \leqslant e^{2 q_{0} C_{0} n}\left(e^{2 q_{0} C_{0} n}+D \rho^{n}\right)\left(\widetilde{\psi}+C_{0} D \rho^{n}\right) .
$$


Let $n_{0}=n_{0}\left(f, \theta, \delta_{0}\right) \geqslant 1$ be the integer such that

$$
e^{-n_{0} \alpha}<\frac{\delta_{0}}{16 C_{0} D} \leqslant e^{-\left(n_{0}-1\right) \alpha}
$$

Then $-n_{0} \alpha<\log \delta_{0} /\left(16 C_{0} D\right) \leqslant-\left(n_{0}-1\right) \alpha$, so $n_{0}$ satisfies (13). With this choice of $n_{0}$ define $q_{0}$ by (12). Then for $q \in\left[0, q_{0}\right]$ we have $12 q C_{0}^{2} n_{0} \leqslant \delta_{0} / 8$ and so $12 q C_{0} n_{0} \leqslant 1$. It now follows from (15) with $q \in\left[0, q_{0}\right]$ and $n=n_{0}, 0<\delta_{0} \leqslant B_{\psi}=$ $\widetilde{\psi} \leqslant C_{0},(16)$ and the fact that $e^{x} \leqslant 1+3 x$ for $x \in[0,1]$ that

$$
\begin{aligned}
\int \psi d \mu_{q} & \leqslant\left(e^{4 q_{0} C_{0} n_{0}}+D e^{2 q_{0} C_{0} n_{0}} e^{-\alpha n_{0}}\right)\left(\widetilde{\psi}+C_{0} D e^{-\alpha n_{0}}\right) \\
& \leqslant\left(1+12 q_{0} C_{0} n_{0}+\left(1+6 q_{0} C_{0} n_{0}\right) \frac{\delta_{0}}{16 C_{0}}\right)\left(\widetilde{\psi}+\frac{\delta_{0}}{16}\right) \\
& \leqslant \widetilde{\psi}+12 q_{0} C_{0}^{2} n_{0}+\left(1+6 q_{0} C_{0} n_{0}\right) \frac{\delta_{0}}{16}+\left(2+2 \frac{\delta_{0}}{16 C_{0}}\right) \frac{\delta_{0}}{16} \\
& \leqslant \widetilde{\psi}+\frac{\delta_{0}}{8}+\frac{\delta_{0}}{8}+\frac{3 \delta_{0}}{16}<\widetilde{\psi}+\frac{\delta_{0}}{2} .
\end{aligned}
$$

Thus, in the case $p \geqslant \widetilde{\psi}+\delta_{0}$, it follows from (11) that $\Gamma^{\prime}(q) \geqslant \delta_{0} / 2$ for all $q \in\left[0, q_{0}\right]$, and therefore $\Gamma\left(q_{0}\right) \geqslant \delta_{0} q_{0} / 2$.

Next, assume that $p \leqslant \widetilde{\psi}-\delta_{0}$. We will now estimate $\int \psi d \mu_{q}$ from below for $q \in\left[-q_{0}, 0\right]$. As in the previous estimate, using (9) and (10), for such $q$ we get

$$
\begin{aligned}
\int \psi d \mu_{q} & =\int \psi h_{q} d v_{q}=\frac{1}{\lambda_{q}^{n_{0}}} \int L_{q}^{n_{0}}\left(\psi h_{q}\right) d v_{q} \\
& =\frac{1}{\lambda_{q}^{n_{0}}} \int \sum_{\sigma^{n_{0} y=x}} e^{(\phi+q \psi)_{n_{0}}(y)} \psi(y) h_{q}(y) d v_{q}(x) \\
& \geqslant e^{-2 q_{0} C_{0} n_{0}}\left(e^{-2 q_{0} C_{0} n_{0}}-D \rho^{n_{0}}\right) \int L^{n_{0}} \psi d v_{q}
\end{aligned}
$$

Notice that by the choice of $q_{0}$ and $n_{0}$ we have $e^{-2 q_{0} C_{0} n_{0}}-D \rho^{n_{0}}>0$. In fact, it follows from $e^{-x}>1-x$ for $x>0$ that $e^{-2 q_{0} C_{0} n_{0}}>1-2 q_{0} C_{0} n_{0}$, while (16) implies $D \rho^{n_{0}}<\delta_{0} /\left(16 C_{0}\right)$. Thus,

$$
e^{-2 q_{0} C_{0} n_{0}}-D \rho^{n_{0}}>1-2 q_{0} C_{0} n_{0}-\frac{\delta_{0}}{16 C_{0}}>1-\frac{\delta_{0}}{8 C_{0}} .
$$

On the other hand, (14) yields $\int L^{n_{0}} \psi d v_{q} \geqslant \widetilde{\psi}-D C_{0} \rho^{n_{0}}>\widetilde{\psi}-\delta_{0} / 16$. Hence for $q \in\left[-q_{0}, 0\right]$ we get 


$$
\begin{aligned}
\int \psi d \mu_{q} & \geqslant\left(1-2 q_{0} C_{0} n_{0}\right)\left(1-\frac{\delta_{0}}{8 C_{0}}\right)\left(\widetilde{\psi}-\frac{\delta_{0}}{16}\right) \\
& \geqslant\left(1-2 q_{0} C_{0} n_{0}-\frac{\delta_{0}}{8 C_{0}}\right)\left(\widetilde{\psi}-\frac{\delta_{0}}{16}\right) \\
& \geqslant \widetilde{\psi}-\widetilde{\psi}\left(2 q_{0} C_{0} n_{0}+\frac{\delta_{0}}{8 C_{0}}\right)-\frac{\delta_{0}}{16} \geqslant \widetilde{\psi}-\frac{\delta_{0}}{50}-\frac{\delta_{0}}{8}-\frac{\delta_{0}}{16}>\widetilde{\psi}-\frac{\delta_{0}}{2} .
\end{aligned}
$$

Thus, for $q \in\left[-q_{0}, 0\right]$ we have

$$
\Gamma^{\prime}(q)=p-\int \psi d \mu_{q} \leqslant \widetilde{\psi}-\delta_{0}-\left(\widetilde{\psi}-\delta_{0} / 2\right) \leqslant-\frac{\delta_{0}}{2},
$$

and therefore $\Gamma\left(-q_{0}\right) \geqslant \delta_{0} q_{0} / 2$.

Proof of Theorem 1.1 Assume again that $\psi \geqslant 0$. Let $p \geqslant \widetilde{\psi}+\delta_{0}$. Then $I(p)=$ $\sup _{q \in \mathbb{R}} \Gamma(q)$, so by Lemma $2.3, I(p) \geqslant \Gamma\left(q_{0}\right) \geqslant \delta_{0} q_{0} / 2$. Similarly, for $p \leqslant \widetilde{\psi}-\delta_{0}$ we get $I(p) \geqslant \delta_{0} q_{0} / 2$.

As explained in Sect. 2.2, the case of an arbitrary real-valued $\psi \in \mathcal{F}_{\theta}\left(\Sigma_{A}^{+}\right)$follows from the case $\psi \geqslant 0$.

Acknowledgments Thanks are due to the referees for their valuable comments and suggestions.

\section{References}

1. Bowen, R.: Equilibrium States and the Ergodic Theory of Anosov Diffeomorphisms. Lecture Notes in Mathematics, vol. 470. Springer, Berlin (1975)

2. Kachurovskii, A.G., Podvigin, I.V.: Large deviations and rates of convergence in the Birkhoff ergodic theorem: from Hölder continuity to continuity. Dokl. Math. 93(1), 6-8 (2016)

3. Kifer, Yu.: Large deviations in dynamical systems and stochastic processes. Trans. Amer. Math. Soc. 321(2), 505-524 (1990)

4. Lalley, S.P.: Distribution of periodic orbits of symbolic and Axiom A flows. Adv. in Appl. Math. 8(2), 154-193 (1987)

5. Leplaideur, R., Saussol, B.: Large deviations for return times in non-rectangle sets for Axiom A diffeomorphisms. Discrete Contin. Dyn. Syst. 22(1-2), 327-344 (2008)

6. Orey, S., Pelikan, S.: Deviations of trajectory averages and the defect in Pesin's formula for Anosov diffeomorphisms. Trans. Amer. Math. Soc. 315(2), 741-753 (1989)

7. Parry, W., Pollicott, M.: Zeta Functions and the Periodic Orbit Structure of Hyperbolic Dynamics. Astérisque, vol. 187-188. Société Mathmatique de France, Paris (1990)

8. Petkov, V., Stoyanov, L.: Sharp large deviations for some hyperbolic systems. Ergodic Theory Dynam. Systems 35(1), 249-273 (2015)

9. Pollicott, M., Sharp, R.: Large deviations, fluctuations and shrinking intervals. Comm. Math. Phys. 290(1), 321-334 (2009)

10. Ruelle, D.: Thermodynamic Formalism. Encyclopedia of Mathematics and its Applications, vol. 5. Addison-Wesley, Reading (1978)

11. Stoyanov, L.: On the Ruelle-Perron-Frobenius theorem. Asymptot. Anal. 43(1-2), 131-150 (2005)

12. Young, L.-S.: Large deviations in dynamical systems. Trans. Amer. Math. Soc. 318(2), 525-543 (1990) 